K. SHIGA

KODAI MATH. J.

6 (1983), 220-227

\title{
DISCRETE STRUCTURES AND VAN DER WAERDEN SETS
}

\author{
By KojI SHIgA
}

In a previous paper [2], we introduced a notion on discrete structure on a compact smooth manifold $M$. Actually, a discrete structure is to give an equivalence class of everywhere dense sequences on $M$, where two such sequences $\left\{a_{n}\right\}$ and $\left\{b_{n}\right\}$ are called equivalent if there are diffeomorphism $\varphi$ of $M$ and a positive integer $N$ such that

$$
\varphi\left(a_{n}\right)=b_{n} \quad \text { for } \quad n \geqq N .
$$

In the present paper, we attach a closed set of $M$ to an everywhere dense sequence $\left\{a_{n}\right\}$ of $M$, called van der Waerden set, which consists of those points that any neighborhood of the point contains arbitrarily long subsequence of $\left\{a_{n}\right\}$ with the form $\left\{a_{s}, a_{s+r}, a_{s+2 r}, \cdots, a_{s+l r}\right\}$. Then any everywhere dense sequence has a non-empty van der Waerden set, while for any closed set $F$ of $M$ we can find an everywhere dense sequence whose van der Waerden set coincides with $F$. Also the homeomorphism type of van der Waerden set gives an invariant of discrete structures.

We note that, while the notion of van der Waerden sets basically lies on toplogical level, our investigations are concerned with smooth manifolds. We have much interest in studying the way of distributions of tangent vectors associated to dense sequences. In this view, we introduce a notion of van der Waerden set $W(\boldsymbol{a}, \boldsymbol{b})$ defined for two everywhere dense sequences $\boldsymbol{a}=\left\{a_{n}\right\}$ and $\boldsymbol{b}=\left\{b_{n}\right\}$ on $M$, close to each other. This $W(\boldsymbol{a}, \boldsymbol{b})$ is a closed set of the tangent sphere bundle of $M$, on which the behavior of the recurrent properties of the direction vectors directed from $a_{n}$ to $b_{n}(n=1,2, \cdots)$ reflects. We will establish some elementary properties on $W(\boldsymbol{a}, \boldsymbol{b})$.

1. Let $S$ be a subset of $N$, where $N$ denotes the set of positive integers. Putting

$$
B D^{*}(S)=\lim _{I} \sup \frac{|S \cap I|}{|I|},
$$

we call it the upper Banach density of $S$, where $I$ ranges over all intervals of $N$. More precisely, if $B D^{*}(S)=d$, then there is a sequence $\left\{I_{n}\right\}$ with $\left|I_{n}\right| \rightarrow \infty$, for which we have

$$
\lim \frac{\left|S \cap I_{n}\right|}{\left|I_{n}\right|} \rightarrow d
$$

while, for any sequence $\left\{J_{n}\right\}$ with $\left|J_{n}\right| \rightarrow \infty$

Received September 6, 1982 


$$
\lim \sup \frac{\left|S \cap J_{n}\right|}{\left|J_{n}\right|} \leqq d .
$$

Then it is clear that, for any subset $S_{1}$ and $S_{2}$ of $N$, we have

$$
B D^{*}\left(S_{1} \cup S_{2}\right) \leqq B D^{*}\left(S_{1}\right)+B D^{*}\left(S_{2}\right)
$$

and, if $B D^{*}\left(S_{2}\right)=0$, we have

$$
B D^{*}\left(S_{1} \cup S_{2}\right)=B D^{*}\left(S_{1}\right) .
$$

Also, it is not difficult to show that, for any $0 \leqq \alpha \leqq 1$, there is a subset $S$ of $N$ with $B D^{*}(S)=\alpha$.

Let $M$ be a compact smooth manifold. Let $\boldsymbol{a}=\left\{a_{n}\right\}$ be an everywhere dense sequence on $M$, where we always assume $a_{m} \neq a_{n}$ for $m \neq n$. For any open set $V$ of $M$, set $S_{V}=\left\{n \mid a_{n} \in V\right\}$ and define

$$
B D_{\boldsymbol{a}}^{*}(V)=B D^{*}\left(S_{V}\right) .
$$

We have then $0 \leqq B D_{\boldsymbol{a}}^{*}(V) \leqq 1$, and if $U \subset V$, we have $B D_{\boldsymbol{a}}^{*}(U) \leqq B D^{*}(V)$. Put

$$
d_{\boldsymbol{a}}(x)=\lim B D_{\boldsymbol{a}}^{*}(V(x)),
$$

where $V(x)$ ranges over a basis of open neighborhoods of $x$.

Definition 1. $d_{\boldsymbol{a}}(x)$ is called density at $x$ associated to $\boldsymbol{a}$.

LEMMA 1. If $x_{n} \rightarrow x_{0}$, then $\lim \sup d_{\boldsymbol{a}}\left(x_{n}\right) \leqq d_{\boldsymbol{a}}\left(x_{0}\right)$.

This is clear from the definition. Hence $d_{\boldsymbol{a}}(x)$ gives an upper semi-continuous function on $M$. It follows that the way of value-distribution of $d_{\boldsymbol{a}}(x)$ is not so arbitrary. However, we have

THEOREM 1. Let $F$ be a non-empty closed set of $M$. Then there exists an everywhere dense sequence $\boldsymbol{a}=\left\{a_{n}\right\}$ with the property that

$$
d_{a}(x)=\left\{\begin{array}{lll}
1 & \text { if } & x \in F, \\
0 & \text { if } & x \notin F .
\end{array}\right.
$$

The next lemma may be interesting in itself.

LEMMA 2. Let $\left\{a_{n}\right\}(n=1,2, \cdots)$ be an everywhere dense sequence on $M$. Then there exists a smooth triangulation $\Delta$ of $M$ with the following property. Let

$$
\Delta=\Delta^{(1)}, \Delta^{(2)}, \cdots, \Delta^{(l)}, \cdots
$$

be a sequence of triangulations of $M$ which are obtained by successive barycentruc subdivisions of $\Delta$. Then each $a_{n}$ is contained in the interior of some $k$-simplex at every triangulation $\Delta^{(l)}(l=1,2, \cdots)$, where $k=\operatorname{dim} M$. 
Proof. Take any smooth triangulation $\tilde{\Delta}$ of $M$ and consider a sequence of successive barycentric subdivisions of $\Delta$ :

$$
\tilde{\Delta}=\tilde{\Delta}^{(1)}, \tilde{\Delta}^{(2)}, \cdots, \tilde{\Delta}^{(l)}, \cdots .
$$

Let $Q$ be the union of the faces of all the simplices arising from $\tilde{\Delta}^{(l)}(l=1,2, \cdots)$. Then $Q$ has measure 0 , whence we can take an everywhere dense sequence $\left\{b_{n}\right\}$ $(n=1,2, \cdots)$ from the outside of $Q$. By Proposition 1 of $[2]$, we know that there is a diffeomorphism $\varphi$ of $M$ such that

$$
\varphi\left(\cup b_{n}\right)=\cup a_{n} .
$$

Then, putting $\Delta=\varphi(\tilde{\Delta})$, we find that $\Delta$ satisfies the required property.

Proof of theorem. We assume that $F$ is an infinite set. The case where $F$ is a finite set will be proved in a similar way. Let $N$ devide in a countable family of infinite subsets such that

$$
N=J_{1} \sqcup J_{2} \sqcup \cdots \sqcup J_{s} \sqcup \cdots \sqcup \sqcup,
$$

where each $J_{s}(s=1,2, \cdots)$ has density 1 and $J$ has density 0 . Let $J_{s}=\left\{n_{s, 1}, n_{s, 2}\right.$, $\left.n_{s, 3}, \cdots\right\}$ and $J=\left\{n_{1}, n_{2}, \cdots\right\}$. We take an everywhere dense countable subset $S$ of $M$ and arrange the points of $S$ in order, using $J$ as the set of indices, so that

$$
S=\left\{a_{n_{1}}, a_{n_{2}}, \cdots\right\} .
$$

Choose an everywhere dense sequence $\left\{x_{s}\right\}(s=1,2, \cdots)$ from $F$. We add a countable set $\left\{y_{t}\right\}(t=1,2, \cdots)$ to $\left\{x_{s}\right\}$ in order to get an everwhere dense set $\left\{x_{s}, y_{t}\right\} \quad(s, t=1,2, \cdots)$ on $M$, and apply Lemma to this sequence $\left\{x_{s}, y_{t}\right\}$.

As a result, we find that there is a smooth triangulation $\Delta$ of $M$ such that, in the sequence

$$
\Delta=\Delta^{(1)}, \Delta^{(2)}, \cdots, \Delta^{(l)}, \cdots
$$

of barycentric subdivisions of $\Delta$, each $x_{s}$ is contained in the interior of some $k$ dimensional simplex, say $\Delta_{s}^{(l)}$, at each $\Delta^{(l)}(k=\operatorname{dim} M)$. Hence $\left\{\Delta_{s}^{(l)} \mid l=1,2, \cdots\right\}$ gives a sequence of $k$-simplices tending to $x_{s}$. Take any point $p$ from $\Delta_{s}^{(l+s)}$ and assign a number $n_{s, l}$ of $J_{s}$ to it. In such a way, we have a point $p=a_{n_{s, l}}$ in ${\Delta_{s}(l+s)}^{(l+}$. We may and do assume each $a_{n_{s, l}}(s, l=1,2, \cdots)$ is distinct. We have thus for each $s$ a sequence of points $\left\{a_{n_{s, l}} \mid l=1,2, \cdots\right\}$ tending to $x_{s}$, whose set of indices is given by the subset $J_{s}$ of $N$ with density 1 . Also, $a_{n_{s}, l}$ will be contained in a smaller and smaller simplex when $s \rightarrow \infty$.

Combining the sequence $\left\{a_{n_{s, l}} \mid s, l=1,2, \cdots\right\}$ with (1), we have an everywhere dense sequence

$$
\boldsymbol{a}=\left\{a_{1}, a_{2}, a_{3}, \cdots\right\}
$$

of $M$. From the construction, it follows that $d_{\boldsymbol{a}}\left(x_{s}\right) \geqq 1$, or, what amounts to the same thing, $d_{a}\left(x_{s}\right)=1$ for $s=1,2, \cdots$. Since $\left\{x_{s}\right\}$ is dense in $F$, by Lemma 1 we can conclude $d_{a}(x)=1$ for $x \in F$. On the other hand, take $y$ from the out- 
side of $F$. Then, if we take a small neighborhood $V(y)$ of $y, V(y)$ contains no point of $\left\{a_{n_{s, l}}\right\}$ except a finite number of $\left\{a_{n_{s, l}}\right\}$, from which follows directly $d_{\boldsymbol{a}}(y)=0$. This completes the proof.

We note that $F$ is obtained by the completion of the Cauchy sequences

$$
\rho\left(a_{n_{i}}, a_{n_{j}}\right) \longrightarrow 0 \quad(\imath, \jmath \rightarrow \infty, \rho: \text { distance function })
$$

where the set of indices $\left\{n_{i}\right\}$ belongs to the set with density 1 . This situation may be well expressed that $F$ is obtained from $\boldsymbol{a}$ by the completion of Cauchy sequences in density 1.

2. We first recall the famous theorem due to van der Waerden.

If the set $N$ is partitioned in a finite family of subsets, then at least one subset contains arbitrarily long arithmetic progression.

In 1975, E. Szemerédi [3] succeeded in obtaining an extension of the above theorem.

If a subset $S$ of $N$ has positive upper Banach density, then $S$ contatins arbitrarily long arithmetic progression.

Let an everywhere dense sequence $\boldsymbol{a}=\left\{a_{n}\right\}$ be given on $M$.

Definition 2. If a point $p$ of $M$ satisfies the following condition, then we say that $p$ is a van der Waerden point of $\boldsymbol{a}$ :

Any neighborhood of $p$ contains a finite subsequence of $\boldsymbol{a}$ with the form $\left\{a_{s}, a_{s+k}, \cdots, a_{s+l k}\right\}$, where the length $l$ may be arbitrarily long.

The set consisting of van der Waerden points of $\boldsymbol{a}$ is called the van der Waerden set associated to $\boldsymbol{a}$.

We denote by $W(\boldsymbol{a})$ the van der Waerden set associated to $\boldsymbol{a}$. Intuitively, a van der Waerden point $p$ is interpreted as a kind of recurrent point in the sense that the points of the sequence $\left\{a_{1}, a_{2}, \cdots\right\}$ appear arbitrarily many times (finite!) with a period in any small neighborhood of $p$.

THEOREM 2. (i) For any $\boldsymbol{a}=\left\{a_{n}\right\}, W(\boldsymbol{a})$ is a non-empty closed set of $M$.

(ii) For any non-empty closed set $F$ of $M$, there exists $\boldsymbol{a}=\left\{a_{n}\right\}$ with $W(\boldsymbol{a})=F$.

Proof of (i). We take a smooth triangulation $\Delta$ of $M$ and then consider a sequence of successive barycentric subdivisions of $\Delta$ :

$$
\Delta=\Delta^{(1)}, \Delta^{(2)}, \cdots, \Delta^{(l)}, \cdots .
$$

If we abuse the terminology $k$-simplex for 'half open $k$-simplex' (corresponding to a half open interval as 1-dimensional case), we may assume that each $k$ simplex belonging to $\Delta^{(l)}(l=1,2, \cdots)$ is disjoint to each other. Let

$$
\Delta_{1}^{(1)}, \Delta_{2}^{(1)}, \cdots, \Delta_{s_{1}}^{(1)}
$$

be the $k$-simplices belonging to $\Delta^{(1)}$. Then for some $i_{1}\left(1 \leqq i_{1} \leqq s_{1}\right)$ 


$$
S_{i_{1}}^{(1)}=\left\{n \mid a_{n} \in \Delta_{i_{1}}^{(1)}\right\}
$$

has positive upper Banach density. Let

$$
\Delta_{\jmath_{1}}^{(2)}, \cdots, \Delta_{\jmath_{r}}^{(2)}
$$

be $k$-simplices of $\Delta^{(2)}$ which are subdivisions of $\Delta_{i_{1}}^{(1)}$. Then for some $i_{2}$

$$
S_{\imath_{2}}^{(2)}=\left\{n \mid a_{n} \in \Delta_{\imath_{2}}^{(2)}\right\}
$$

has positive upper Banach density. This process can be carried on at each stage of subdivisions. Hence we have a decreasing sequence of $k$-simplices

$$
\Delta_{i_{1}}^{(1)} \supset \Delta_{i_{2}}^{(2)} \supset \cdots \supset \Delta_{\imath_{l}}^{(l)} \supset \cdots
$$

and a decreasing subsets of $N$

$$
S_{i_{1}}^{(1)} \supset S_{\imath_{2}}^{(2)} \supset \cdots \supset S_{\imath_{l}}^{(l)} \supset \cdots
$$

each of which has positive upper Banach density. Put $p_{0}=\cap S_{\imath}^{(l)}$. Then in view of Szemerédi's theorem, we find that $p_{0}$ is a van der Waerden point of $\boldsymbol{a}$. Hence $W(\boldsymbol{a}) \neq \varnothing$. On the other hand, from the definition it is clear that $W(\boldsymbol{a})$ is closed. This completes the proof.

Proof of (ii). By virtue of Theorem 1, we can take an everywhere dense sequence $\boldsymbol{a}=\left\{a_{n}\right\}$ such that $d_{\boldsymbol{a}}(x)=1$ on $F$ and $=0$ outside $F$. Here we may assume that the subset $J$ of $N$ which appears in the proof of Theorem 1 contains no three integers which form arithmetic progression. Then by Szemerédi's Theorem we find that this $\boldsymbol{a}$ satisfies the desired property, which completes the proof.

The following proposition gives an example of $W(\boldsymbol{a})=M$.

Proposition 1. Let $\varphi$ be a diffeomorphism of $M$ such that, for any point $p$ of $M$,

$$
\bigcup_{n=1}^{\infty} \varphi^{n}(p)
$$

forms an everywhere dense set of $M$. Take any point $p_{0}$ and put $\boldsymbol{a}=\left\{a_{n}\right\}$, where $a_{n}=\varphi^{n}\left(p_{0}\right)$. Then we have $W(\boldsymbol{a})=M$.

Proof. Let $q_{0}$ be a van der Waerden point of $\boldsymbol{a}$. This implies that in any neighborhood $V\left(q_{0}\right)$ of $q_{0}$ there exists a finite sequence

$$
\varphi^{s}\left(p_{0}\right), \varphi^{s+r}\left(p_{0}\right), \cdots, \varphi^{s+l r}\left(p_{0}\right),
$$

where $l$ is any given positive integer. Hence, for any $n$, the neighborhood $\varphi^{n}\left(V\left(q_{0}\right)\right)$ of $\varphi^{n}\left(q_{0}\right)$ contains

$$
\varphi^{n+s}\left(p_{0}\right), \varphi^{n+s+r}\left(p_{0}\right), \cdots, \varphi^{n+s+l r}\left(p_{0}\right),
$$


which shows that $\varphi^{n}\left(q_{0}\right)$ is a van der Waerden point. Since $\bigcup_{n=1}^{\infty} \varphi^{n}\left(q_{0}\right)$ is dense, it follows that $W(\boldsymbol{a})=M$.

Finally, we state a proposition, which shows a relation of van der Waerden set with discrete structures.

Proposition 2. Let $\boldsymbol{a}=\left\{a_{n}\right\}$ and $\boldsymbol{b}=\left\{b_{n}\right\}$ be everywhere dense sequence on $M$. Suppose that $\boldsymbol{a}$ and $\boldsymbol{b}$ give the same discrete structure to $M$. Then $W(\boldsymbol{a})$ is diffeomorphic to $W(\boldsymbol{b})$ through a diffeomorphism of $M$.

The proof is immediate.

3. In the above discussions, we have not essentially used the fact that the sequence $\boldsymbol{a}=\left\{a_{n}\right\}$ spreads all over $M$. Hence, in the following rather special situation, the results above will be generalized to be valid. We endow $M$ with a Riemannian metric and consider the tangent sphere bundle $S(M)$ of $M$. Denote by $\pi$ the projection from $S(M)$ to $M$. Let $L$ be a closed set of $S(M)$ with $\pi(L)=M$. If we have an everywhere dense sequence $\Xi=\left\{\xi_{n}\right\}$ on $L$, then, for this $E$, we will be able to obtain the results corresponding to Thorems 1 and 2 relative to $L$ by the similar arguments. Really, let $W_{L}(\boldsymbol{\Xi})$ be the van der Waerden set associated to $\Xi$, which is defined similarly to Definition 2 by using the relative topology of $L$. Hence $W_{L}(\Xi)$ becomes a closed set of $L$. From the definition it is clear that $W_{L}(\boldsymbol{E})$ is disjoint from the isolated points of $L$. Then we have

Proposition 3. (i) $W_{L}(\boldsymbol{\Xi}) \neq \varnothing$.

(ii) Let $F$ be a non-empty closed set of $L$ which contains no isolated pornts of $L$. Then there exists $\boldsymbol{\Xi}=\left\{\xi_{n}\right\}$ with $W_{L}(\boldsymbol{\Xi})=F$.

We will relate this proposition with the discrete structures on $M$. Let $\boldsymbol{a}$ $=\left\{a_{n}\right\}$ and $\boldsymbol{b}=\left\{b_{n}\right\}$ be two everywhere dense sequences on $M$. We assume that $a_{n} \neq b_{n}$ for each $n$ and that $\boldsymbol{b}$ is close to $\boldsymbol{a}$ in the following sense: For each $n$, there exists a unique geodesic $\gamma_{n}(t)$ with $\gamma_{n}(0)=a_{n}, \gamma_{n}(1)=b_{n}$, the length of which attains the distance from $a_{n}$ to $b_{n}$. Put

$$
\xi_{n}=\frac{1}{\left\|\gamma_{n}^{\prime}(0)\right\|} \gamma_{n}^{\prime}(0)
$$

and $\boldsymbol{E}=\left\{\xi_{n}\right\}$. Then $L=\bar{\Xi}$ is a closed set of $S(M)$ with $\pi(L)=M$, and $\boldsymbol{\Xi}$ is an everywhere dense sequence of $L$ with the property

$$
\pi\left(\xi_{m}\right) \neq \pi\left(\xi_{n}\right) \quad \text { if } \quad m \neq n .
$$

We denote the sequence $\Xi$ thus obtained by $D(a, b)$. Conversely, if a dense sequence $\Xi=\left\{\xi_{n}\right\}$ on a closed set $L$ of $S(M)$ with $\pi(L)=M$ satisfies the condition (2), we can find $\boldsymbol{a}$ and $\boldsymbol{b}$ such that $\Xi=D(\boldsymbol{a}, \boldsymbol{b})$. 
DEFInition 3. The van der Waerden set $W_{L}(D(\boldsymbol{a}, \boldsymbol{b}))$ is called the difference van der Waerden set of $\boldsymbol{a}$ and $\boldsymbol{b}$ and is denoted by $W(\boldsymbol{a}, \boldsymbol{b})$.

In view of the restricted condition (2), Proposition 3(ii) cannot be directly applied to the case where $\Xi=D(\boldsymbol{a}, \boldsymbol{b})$. But, in essense, Proposition 3 remains still valid in this case. That is, we have the following theorem. $\neq \varnothing$.

THEOREM 3. (i) For any $\boldsymbol{a}=\left\{a_{n}\right\}$ and $\boldsymbol{b}=\left\{b_{n}\right\}$ close to $\boldsymbol{a}$, we have $W(\boldsymbol{a}, \boldsymbol{b})$

(ii) For any closed set $F$ of $S(M)$, we can find $\boldsymbol{a}$ and $\boldsymbol{b}$ with $W(\boldsymbol{a}, \boldsymbol{b})=F$.

In fact, in order to prove (ii), we apply the similar arguments to those used in the proof of Theorems 1 and 2. In this procedure, we have only to check the fact that we can choose a sequence $\left\{\eta_{n}\right\}$ satisfying the condition (2) which accumulates an everywhere dense set of $F$. But this is easily verified, which will complete the proof.

We note that we have always

$$
\pi(W(\boldsymbol{a}, \boldsymbol{b})) \subset W(\boldsymbol{a}) .
$$

This follows immediately from the definition. Hence, for any given $\boldsymbol{a}, W(\boldsymbol{a}, \boldsymbol{b})$ is not chosen so arbitrarily. However, we do not know what kind of closed set $F$ of $S(M)$ with $\pi(F) \subset W(\boldsymbol{a})$ is expressed as $F=W(\boldsymbol{a}, \boldsymbol{b})$ for a certain $\boldsymbol{b}$.

Let $\boldsymbol{a}=\left\{a_{n}\right\}$ and $\boldsymbol{b}=\left\{b_{n}\right\}$ be two everywhere dense sequences on $M$. Let $\varphi \in \operatorname{Diff}(M)$. The following statement is clear.

Proposition 4. If $W(\boldsymbol{a}, \boldsymbol{b})$ and $W(\varphi(\boldsymbol{a}), \varphi(\boldsymbol{b}))$ are both defined, then $W(\boldsymbol{a}, \boldsymbol{b})$ and $W(\varphi(\boldsymbol{a}), \varphi(\boldsymbol{b}))$ are diffeomorphic via a map $d \varphi$. Here $W(\varphi(\boldsymbol{a}), \varphi(\boldsymbol{b}))$ is defined by the Riemannan metric which is induced by $\varphi$ from the one used in the defintion of $W(\boldsymbol{a}, \boldsymbol{b})$.

It seems to be interesting to clarify the relation between the homeomorphism type of $W(\boldsymbol{a}, \boldsymbol{b})$ and the dependence on Riemannian metric being used for the definition $W(\boldsymbol{a}, \boldsymbol{b})$.

EXAMPLE (as a problem). Let $S^{k}$ be the $k$-dimensional standard sphere. Let $\boldsymbol{a}=\left\{a_{n}\right\}$ be an everywhere dense sequence on $S^{k}$. We suppose that, if $m \neq n, a_{m}$ is not the antipodal point of $a_{n}$. Hence there is a unique geodesic through $a_{m}$ and $a_{n}$. Let $T_{h}(h=1,2, \cdots)$ be the shift transformation of $\boldsymbol{a}=\left\{a_{n}\right\}$. That is,

$$
T_{h} \boldsymbol{\alpha}=\left\{a_{n+h}\right\} .
$$

It is evident that $W\left(T_{h} \boldsymbol{a}\right)=W(\boldsymbol{a})$. Then we can obtain the difference van der Waerden set for each $h$ :

$$
W\left(\boldsymbol{a}, T_{h} \boldsymbol{a}\right), \quad h=1,2, \cdots .
$$

Although the mutual relations of these sets seem to be complicated, we conjecture 
that there might arise some recurrent properties in these sets.

\section{REFERENCES}

[1] H. Furstenberg, Recurrence in ergodic theory and combinatorial number theory, Princeton University Press, 1981.

[2] K. SHIGA, Manifolds and discrete structures, Kodai Math. J., 6 (1983), 204-219.

[3] E. SZEMERÉDI, On sets of integers containing no $k$ elements in arithmetic progression, Acta Arith. 27 (1975), 199-245.

Department of Mathematics

TOKyo Institute of TECHNOLOGy

Oh-Okayama, Meguro

TOKYO, 152

JAPAN 